ADDENDUM TO

\title{
FINITENESS THEOREMS FOR DISCRETE SUBGROUPS OF BOUNDED COVOLUME IN SEMI-SIMPLE GROUPS
}

\author{
by ARmand BOREL and Gopal PRASAD
}

(Publ. Math. I.H.E.S., 69 (1989), 119-171)

1. If $\mathrm{G}$ is an anisotropic outer form of type $\mathrm{A}$, defined over a global function field $k$, then there is a separable quadratic extension $\ell$ of $k$ and a central division algebra $\mathscr{D}$, over $\ell$, with an involution $\sigma$ of the second kind such that either

$$
\mathrm{G}(k)=\left\{d \in \mathscr{D}^{\times} \mid d \sigma(d)=1 \text { and } \operatorname{Nrd}(d)=1\right\}
$$

or there exists an anisotropic hermitian form $h$ on $\mathscr{D}^{2}$, defined in terms of the involution $\sigma$, such that $\mathrm{G}=\mathrm{SU}(h)$. Due to an oversight, in the proof of Theorem 7.3 of [1] the groups $k$-isomorphic to $\mathrm{SU}(h)$, where $h$ is as above, were not considered. The following construction shows that in fact these groups need to be excluded from the purview of the theorem.

Let $q$ be a positive integral power of an odd prime and $\mathbf{F}_{q}$ be the field with $q$ elements. Let $k=\mathbf{F}_{q}(t)$ and $\ell=\mathbf{F}_{q}\left(t^{1 / 2}\right)$. We fix a place $v$ of $k$ which splits over $\ell$ and which is such that the residue field of $k_{v}$ is the field with $q$ elements. We also fix two places $v_{1}, v_{2}$ of $k$, which ramify over $\ell$, and where the residue fields are of cardinality $q$. Now let $\mathscr{D}$ be the central division algebra over $\ell$, of degree $r$, whose local invariant at one of the two places lying over $v$ is $m / r$, at the other place lying over $v$ it is $-m / r$, where $m$ is an integer prime to $r$, and zero at all other places of $\ell$. Let $\sigma$ be an involution of $\mathscr{D}$, of second kind, which fixes $k$ pointwise. Let $h$ be an anisotropic hermitian form on $\mathscr{D}^{2}$, defined in terms of the involution $\sigma$, and $\mathrm{G}=\mathrm{SU}(h)$. We assume that $h$ is so chosen that $\mathrm{G}$ is quasisplit at every place, different from $v_{1}, v_{2}$, which splits over $\ell$, and of rank $r-1$ over $k_{v_{i}}$ for $i=1$, 2. Such a hermitian form exists: in fact, given any $\delta \in k^{\times}$such that $(-1)^{\boldsymbol{\rho}} \delta$ is a norm from $\ell \otimes_{k} k_{w}$ at all places $w$ of $k$ except $v_{1}$ and $v_{2}$, any hermitian form on $\mathscr{D}^{2}$ whose determinant is $\delta$ will have the desired property. (See [5: Chapter 10, Theorem 6.9].)

Both $k$ and $\ell$ are fields of genus zero and

$$
\zeta_{k}(s)=\left(1-q^{-8}\right)^{-1} \cdot\left(1-q^{1-8}\right)^{-1}=\zeta_{\ell}(s) .
$$


Hence, for all $s$,

$$
\mathrm{I}_{\iota / k}(s)=1 \text {. }
$$

$\mathrm{G} / k_{\mathrm{v}}$ is of rank 1 : in fact, there is a central division algebra $\mathrm{D}_{\mathrm{v}}$ over $k_{v}$, of degree $r$ and invariant $m / r$, such that $\mathrm{G}\left(k_{v}\right)$ is isomorphic to $\mathrm{SL}_{2}\left(\mathrm{D}_{v}\right)$. Let $\left(\mathrm{P}_{w}\right)$ be a coherent collection of parahoric subgroups such that for every $w, \mathrm{P}_{w}$ is a parahoric subgroup of $\mathrm{G}\left(k_{w}\right)$ of maximum volume, and let $\Gamma=\mathrm{G}(k) \bigcap_{w \neq v} \mathrm{P}_{w}$. Then, in its natural embedding in $\mathrm{G}\left(k_{v}\right), \Gamma$ is a discrete cocompact subgroup of $\mathrm{G}\left(k_{v}\right)$. Using Theorem 3.7 of [4], we find that the volume of $\mathrm{G}\left(k_{\mathrm{v}}\right) / \Gamma$, with respect to the Tits measure on $\mathrm{G}\left(k_{\mathrm{v}}\right)$, is precisely $\tau_{k}(G)$. Thus, if the Weil conjecture on Tamagawa numbers (i.e., the Tamagawa number of any simply connected semi-simple group, defined over a global field, is 1) holds, then for any central division algebra $\mathrm{D}$ over a local field $\mathrm{K}$ of positive characteristic, the group $\mathrm{SL}_{2}(\mathrm{D})$ contains an arithmetically defined cocompact subgroup of covolume 1 .

2. In the finiteness assertions of Theorems 7.3 and 7.8 of [1] about virtually torsion-free arithmetic subgroups $\Gamma^{\prime}$ with $0 \neq\left|\chi\left(\Gamma^{\prime}\right)\right|<c$, we have excluded the groups of type $A_{2}$. We will now show that using some of the results contained in $\S \S 4.6,3.6(1)$ and 2.10 of [1], the finiteness assertions can be proved also for groups of type $\mathbf{A}_{2}$. We shall use the notation of [1].

Using certain observations in $4.6,3.6$ (1) and 2.10 of [1], we conclude that

$$
\begin{aligned}
\left|\chi\left(\Gamma^{\prime}\right)\right| & \geqslant\left[\Gamma^{\prime}: \Lambda^{\prime}\right]^{-1} \prod_{v \in \mathscr{S}_{f}}\left|\mathrm{~W}_{v}\left(\mathbf{q}^{-1}\right)\right|^{-1} \mu_{\mathscr{S}}\left(\mathrm{G}_{\mathscr{S}} / \Lambda\right) \\
& \geqslant \frac{\prod_{v \in \mathbf{v}-\mathbf{s}} \# \Xi_{\mathscr{\vartheta}_{v}}}{\left[\Gamma^{\prime}: \Lambda^{\prime}\right]} \prod_{v \in \mathscr{S}_{f}}\left|\mathrm{~W}_{v}\left(\mathbf{q}^{-1}\right)\right|^{-1} \mu_{\mathscr{S}}\left(\mathrm{G}_{\mathscr{P}} / \Lambda^{m}\right) \\
& \geqslant\left(\# \prod_{v \in \mathscr{S}} \mathrm{C}^{\prime}\left(k_{v}\right) . \# \mathrm{H}^{1}\left(k, \mathrm{G}_{\xi}^{\prime}\right)^{-1} \prod_{v \in \mathscr{S}_{f}}\left|\mathrm{~W}_{v}\left(\mathbf{q}^{-1}\right)\right|^{-1} \mu_{\mathscr{S}}\left(\mathrm{G}_{\mathscr{S}} / \Lambda^{\mathrm{m}}\right) .\right.
\end{aligned}
$$

Now we note that if $G^{\prime}$ is simply connected,

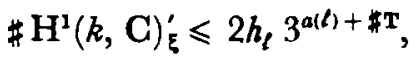

and if $\mathrm{G}^{\prime}$ is adjoint, then

$$
\# H^{1}(k, C)_{\xi}^{\prime} \leqslant 2 h_{l} 3^{a(\ell)+\#\left(8_{f}^{0} \cup T\right)},
$$

where $S_{f}^{0}$ is the subset of places $v \in S_{f}$ such that $G$ splits over $\hat{k}_{v}$ and $T$ is the finite set of places $v(\notin \mathrm{S})$ of $k$ such that $\mathrm{G}$ is anisotropic over $k_{v}$ but splits over $\hat{k}_{v}$. The above bounds follows from the arguments in $[1: \S 5]$ once we observe that if $\mathrm{G}^{\prime}$ is simply connected, $\delta_{v} \varphi^{\prime}\left(\mathrm{G}^{\prime}\left(k_{v}\right)\right)$ is trivial for all $v$, where $\delta_{v}$ is as in $[1: 2.5]$.

Now since

$$
\mu_{\mathscr{S}}\left(G_{\mathscr{S}} / \Lambda^{\mathfrak{m}}\right) \geqslant D_{k}^{\ell}\left(D_{l} / D_{k}^{l \ell: k]}\right)^{5 / 2}\left(\prod_{\bullet \in \mathrm{V}_{\infty}}\left|2^{4} \pi^{5}\right|_{v}^{-1}\right) \tau_{k}(G) \mathscr{E}
$$

where $\sigma$ is an in $[1: 3.8]$, we conclude that

$$
c \geqslant \mathrm{~B}(\mathscr{G} \mid k) \tau \Phi^{\mathrm{RP}} \text {, }
$$


where $\mathrm{B}(\mathscr{G} / k)$ is as in $[1: 7.4(12),(13)]$ and

$$
\Phi^{\mathrm{IPP}}=\prod_{v \in \mathrm{B}_{f}} \varphi_{v} \prod_{v \in \mathbf{v}-\mathbf{s}} f_{v},
$$

for $v \in \mathrm{V}-\mathrm{S}$; here, $f_{v}$ is as in $[1: 7.4]$; for $v \in \mathrm{S}_{f}^{0}$,

$$
\varphi_{v}=3^{-1} e_{v}\left|W_{v}\left(q^{-1}\right)\right|^{-1}
$$

and for $v \in \mathrm{S}_{\rho}-\mathrm{S}_{\rho}^{0}$,

$$
\varphi_{v}=\left(\# \mathrm{C}^{\prime}\left(k_{v}\right)\right)^{-1} \epsilon_{v}\left|\mathrm{~W}_{v}\left(\mathbf{q}^{-1}\right)\right|^{-1} .
$$

We claim that

$$
\left(\mathrm{D}_{\ell} / \mathrm{D}_{k}^{[\ell: k]}\right)^{1 / 2} \prod_{\bullet \in \mathrm{B}_{f}} \varphi_{\vartheta}>1 .
$$

Once the claim is established, Proposition 6.1 (iv), (vi) and the arguments used in the proof of Theorem 7.3 can be employed to establish the finiteness assertions of Theorems 7.3 and 7.8 of [1] for groups of type $A_{2}$ also.

To prove the claim, the following case analysis suffices.

(1) If $\mathrm{G} / k_{\mathrm{v}}$ is of inner type

$$
\varphi_{v}=3^{-1} q_{v}^{s}\left(q_{v}-1\right)\left(q_{v}^{8}-1\right)^{-1}>1
$$

for all $q_{v}$.

(2) If $G / k_{v}$ is an outer form which splits over $\hat{k}_{v}$, then

$$
\varphi_{v}=3^{-1} q_{v}^{5}\left(q_{v}^{2}+1\right)\left(q_{v}^{8}+1\right)^{-1}\left(q_{v}+1\right)^{-1}>1
$$

for all $q_{0}$.

(3) If $\mathrm{G} / k_{\mathrm{v}}$ is an outer form which does not split over any unramified extension of $k_{v}$, then $\left|W_{v}\left(q^{-1}\right)\right|^{-1}=\left(q_{v}-1\right)\left(q_{v}+1\right)^{-1}$, and it is easy to see that $\varphi_{v}>1$ whenever $q_{v}>3$. If $q_{v}=2, \ell_{v}:=\ell \otimes_{k} k_{v}$, being a ramified extension, does not contain a 3rd root of unity, and so $\mathrm{C}^{\prime}\left(k_{v}\right)$ is trivial, $\varphi_{v}=4 / 3$. Finally, if $q_{v}=3$,

$$
\varphi_{v} \geqslant 3^{-1} q_{v}^{2}\left(q_{v}-1\right)^{-1}\left(q_{v}-1\right)\left(q_{v}+1\right)^{-1}=3 / 4>3^{-1 / 2} \text {. }
$$

Now Theorem A of the Appendix of [4] implies the claim.

3. Errata to [1]. We take this opportunity to make the following two corrections in [1]. In the statement of Theorem 7.3, " $\Gamma$ is virtually free" should be replaced by " $\Gamma$ is virtually torsion-free" and in the footnote on $\mathrm{p} .146$ " $\mathrm{G}$ is anisotropic over $k$ if the latter is a number field" should be replaced by " $\mathrm{G}$ is anisotropic over $k$ if the latter is a global function field".

4. It has been pointed out to us by E. A. Nisnevic that Theorem B. 1 of Appendix B of [1] was proved by him in [2] for all connected reductive groups. His method is different 
from the one used in [1]. For the sake of completeness, we present a proof of the more general result in our framework.

Theorem (Borel-Serre, Nisnevic). - Let $\mathrm{G}$ be a connected reductive group defined over a global field $k$. Then the fibers of the canonical map

$$
\lambda_{\mathrm{G}}^{1}: \mathrm{H}^{1}(k, \mathrm{G}) \rightarrow \prod_{v \in \mathbf{v}} \mathrm{H}^{1}\left(k_{v}, \mathrm{G}\right)
$$

are finite.

Let $T$ be the connected component of the identity of the center of $G$ and $G^{\prime}=G / T$. We know that the fibers of the canonical map

$$
\lambda_{\mathrm{G}^{\prime}}^{1}: \mathrm{H}^{1}\left(k, \mathrm{G}^{\prime}\right) \rightarrow \amalg_{v \in \mathrm{V}} \mathrm{H}^{1}\left(k_{v}, \mathrm{G}^{\prime}\right)
$$

are finite [1: Theorem B.1]. Now using the standard twisting trick, it suffices to prove that the subset of $\mathrm{H}^{1}(k, \mathrm{G})$ consisting of the elements which are mapped onto the trivial

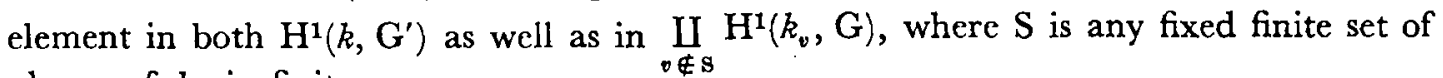
places of $k$, is finite.

Let $\mathrm{S}$ be a finite set of places of $k$ containing all the archimedean places and containing at least one place where $G^{\prime}$ is isotropic. Let $A$ be the ring of adèles of $k$ and $A_{\mathrm{s}}$ be the ring of S-adèles, i.e. the restricted direct product of the $k_{v}$ 's for $v \notin \mathrm{S}$. For every place $v$ of $k$, there is a natural homomorphism $\partial_{v}: \mathrm{G}^{\prime}\left(k_{v}\right) \rightarrow \mathbf{H}^{\mathbf{1}}\left(k_{v}, \mathbf{T}\right)$, and so there is a homomorphism

$$
\partial: \mathrm{G}^{\prime}\left(\mathrm{A}_{\mathrm{g}}\right) \rightarrow \prod_{v \notin \mathrm{S}} \mathrm{H}^{1}\left(k_{\mathrm{v}}, \mathrm{T}\right) .
$$

The kernel of $\partial$ contains a compact open subgroup $\mathrm{K}^{\prime}$ of $\mathrm{G}^{\prime}\left(\mathrm{A}_{\mathrm{g}}\right)$; in fact the image of $G\left(A_{s}\right)$ in $G^{\prime}\left(A_{s}\right)$, under the natural homomorphism $G\left(A_{s}\right) \rightarrow G^{\prime}\left(A_{s}\right)$ is an open subgroup of $G^{\prime}\left(A_{B}\right)$, see, for example, [3:I, 3.6]. In particular, the image of $\partial$ is contained in $\coprod_{v \in \mathrm{B}} \mathrm{H}^{\mathrm{1}}\left(k_{v}, \mathrm{~T}\right)$. Thus we have a commutative diagram with exact rows

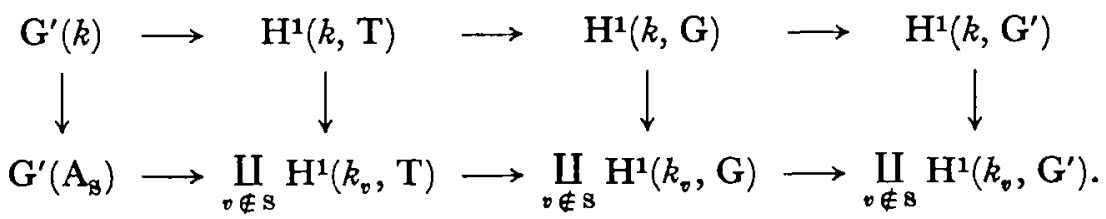

Now the above theorem follows from (1) the finiteness of the kernel of the homomorphism $\mathrm{H}^{1}(k, \mathrm{~T}) \rightarrow \underset{v \notin \mathrm{S}}{\amalg} \mathrm{H}^{1}\left(k_{v}, \mathrm{~T}\right)[3: \mathrm{IV}, 2.6],(2)$ the finiteness of the number of double cosets $\mathrm{G}^{\prime}(k) \backslash \mathrm{G}^{\prime}(\mathrm{A}) /\left(\mathrm{G}_{\mathrm{g}}^{\prime} \mathrm{K}^{\prime}\right)[1$ : Proposition 3.9], and a diagram chase. 


\section{REFERENCES}

[1] A. Borel and G. Prasad, Finiteness theorems for discrete subgroups of bounded covolume in semi-simple groups, Publ. Math. I.H.E.S., 69 (1989), 119-171.

[2] E. A. Nisnevič, Theorem of properness of Galois cohomology maps over function fields (in Russian), Dokl. Acad. Nauk BSSR, 23 (1979), 1065-1068.

[3] J. Oesterlé, Nombres de Tamagawa et groupes unipotents en caractéristique $p$, Invent. Math., 78 (1984), 13-88.

[4] G. PRASAD, Volumes of S-arithmetic quotients of semi-simple groups, Publ. Math. I.H.E.S., 69 (1989), 91-117.

[5] W. Scharlau, Quadratic and hermitian forms, Springer-Verlag, Heidelberg (1985).

Institute for Advanced Study

Princeton N.J. 08540

U.S.A.

Tata Institute of Fundamental Research

Homi Bhabha Road

Bombay 400005

India

Manuscrit rę̧u le 5 juin 1990. 